\title{
Perioperative statin therapy reduces mortality in normolipidemic patients undergoing cardiac surgery
}

\author{
Muthiah Vaduganathan, BA, Neil J. Stone, MD, Richard Lee, MD, MBA, Edwin C. McGee, Jr, MD, \\ S. Chris Malaisrie, MD, Robert A. Silverberg, MD, and Patrick M. McCarthy, MD
}

\begin{abstract}
Objective: Statins might have pleiotropic effects, independent of their ability to reduce lipid levels. Recent data have suggested that statins improve early survival and cardiovascular outcomes after coronary artery bypass graft surgery. The effectiveness of statin therapy in normolipidemic cardiac surgery patients is as yet unclear.

Methods: We evaluated 3056 consecutive patients who had undergone cardiac surgery between April 2004 and April 2009. Perioperative statin therapy was defined as continued treatment both before ( $\geq 6$ months) and after the index surgery (included as a discharge medication). Hyperlipidemia (HL) was defined as a total cholesterol level greater than $200 \mathrm{mg} / \mathrm{dL}$ within 6 months before surgery. Four groups were analyzed: (1) statin-untreated normolipidemic (NL-, $\mathrm{n}=1052)$; (2) statin-treated normolipidemic (NL+, $\mathrm{n}=206)$; (3) statin-untreated hyperlipidemic $(\mathrm{HL}-, \mathrm{n}=638)$; and (4) statin-treated hyperlipidemic $(\mathrm{HL}+, \mathrm{n}=1160)$ patients. Adjusted hazard ratios accounted for the known preoperative cardiac risk factors. Mortality was ascertained by retrospective database review and the Social Security Death Index.
\end{abstract}

Results: The mean follow-up was 2.2 years. The crude rate of 30-day mortality was $3.0 \%(32 / 1052), 0 \%(0 /$ 206), $8.0 \%$ (51/638), and $0.7 \%$ (8/1160) for the NL-, NL+, HL-, and HL+ groups, respectively. The overall all-cause crude mortality rate was $9.6 \%$ (101/1052), 3.9\% (8/206), $17.2 \%(110 / 638)$, and $6.5 \%(75 / 1160)$ for the NL-, NL+, HL-, and HL+ groups, respectively. Statin therapy for NL patients undergoing cardiac surgery independently reduced the overall all-cause mortality (adjusted hazard ratio, 0.34; $95 \%$ confidence interval, $0.16-0.71 ; P=.004)$.

Conclusions: Perioperative statin therapy was associated with reduced mid-term mortality for patients undergoing cardiac surgery, irrespective of their baseline lipid status. This clinical evidence suggests that the beneficial effects of statins might extend beyond their lipid-lowering ability. (J Thorac Cardiovasc Surg 2010;140:1018-27)

Earn CME credits at

http://cme.ctsnetjournals.org

Statins are powerful inhibitors of 3-hydroxyl-3-methylglutaryl-coenzyme A reductase, the key rate-limiting step in cholesterol biosynthesis, thereby enhancing the synthesis of low-density lipoprotein (LDL) receptors and resulting in diminished LDL cholesterol levels. ${ }^{1}$ Their ease of use, potent reduction of apo-B fractions, and proven clinical benefit in a number of primary and secondary clinical trials

\footnotetext{
From the Division of Cardiac Surgery, Bluhm Cardiovascular Institute, Northwestern University Feinberg School of Medicine and Northwestern Memorial Hospital, Chicago, Ill.

Disclosures: None.

Read at the 36th Annual Meeting of The Western Thoracic Surgical Association, Ojai, Calif, June 23-26, 2010.

Received for publication June 25, 2010; revisions received July 28, 2010; accepted for publication Aug 4, 2010; available ahead of print Sept 20, 2010.

Address for reprints: Richard Lee, MD, MBA, Division of Cardiac Surgery, Northwestern University, 201 East Huron Street, Suite 11-140, Chicago, IL 606112908 (E-mail: ricklee@nmh.org).

0022-5223/\$36.00

Copyright $(2010$ by The American Association for Thoracic Surgery doi:10.1016/j.jtcvs.2010.08.002
}

have brought statins to the forefront of current lipidlowering regimens. ${ }^{2,3}$

The National Cholesterol Education Program Adult Treatment Panel III Guidelines ${ }^{4}$ expanded on the appropriate candidates for statin therapy to include high-risk patients with coronary heart disease, acute coronary syndromes, diabetes mellitus, and severe hypercholesterolemia. The current recommendations have supported aggressive reductions in LDL cholesterol in patients with underlying cardiovascular disease or those with coronary equivalents. Statin therapy has become a mainstay in the perioperative treatment of patients undergoing cardiac surgery within the past few years. In a meta-analysis of more than 30,000 patients, Liakopoulos and colleagues ${ }^{5}$ found that preoperative statin therapy significantly reduced early postoperative adverse outcomes in patients undergoing coronary artery bypass grafting (CABG).

To better understand the LDL-dependent and LDLindependent ("pleiotropic") effects of statin therapy, the baseline cholesterol levels must be considered in the context of statin efficacy. Emerging data have pointed to the beneficial effects of statins in patients with hyperlipidemia (HL) undergoing cardiac surgery. Thielmann and colleagues ${ }^{6}$ found that patients with HL who were pretreated with statin therapy were at a significantly lower risk of major early 


$$
\begin{array}{ll}
\text { Abbreviations and Acronyms } \\
\begin{aligned}
\text { CABG } & =\text { coronary artery bypass grafting } \\
\text { CI } & =\text { confidence interval } \\
\text { HL } & =\text { hyperlipidemia } \\
\text { HR } & =\text { hazard ratio } \\
\text { ICU } & =\text { intensive care unit } \\
\text { JUPITER }= & \text { Justification for the Use of Statins in } \\
& \text { Prevention: an Intervention Trial } \\
& \text { Evaluating Rosuvastatin } \\
\text { LDL } & =\text { low-density lipoprotein } \\
\text { LOS } & =\text { length of stay } \\
\text { NL } & =\text { normolipidemia } \\
\text { NYHA } & =\text { New York Heart Association } \\
\text { OR } & =\text { odds ratio }
\end{aligned}
\end{array}
$$

effects of perioperative statin therapy on HL and NL patients undergoing cardiac surgery.

\section{MATERIALS AND METHODS \\ Study Design}

We used a retrospective cohort design and evaluated 3056 consecutive patients (2007 men, 1049 women; aged 18 to 95 years) who had undergone cardiac surgery at Northwestern Memorial Hospital between April 1, 2004 and April 1, 2009. Data regarding statin use was obtained from a prospectively collected in-hospital surgical database; however, the data detailing the dosages and types of statins used were not available. Because the effects of acute changes in statin therapy during the hospital visit are unclear, ${ }^{20}$ we decided to use a more conservative definition of statin therapy. Perioperative statin therapy was defined as continued treatment both before (at least 6 months) and after the index surgery (included as a discharge medication). HL was defined as a total cholesterol level greater than $200 \mathrm{mg} / \mathrm{dL}$ within 6 months before surgery. Using these definitions, 4 groups were analyzed: (1) statin-untreated NL $(\mathrm{NL}-, \mathrm{n}=1052)$; (2) statin-treated NL (NL+, $\mathrm{n}=206)$; (3) statin-untreated HL (HL-, $\mathrm{n}=638)$; and (4) statin-treated HL (HL+, $\mathrm{n}=1160)$ patients. The institutional review board of Northwestern Memorial Hospital reviewed and approved the present study. All patients included in the study provided explicit informed consent for the use of their medical records for research purposes.

\section{Baseline Covariates}

A retrospective database review was used to document the patient baseline characteristics, including demographics (age, gender, race); clinical characteristics (body mass index, current smoking status, severe comorbidities, personal or family history of cardiovascular disease); surgical characteristics (ejection fraction, preoperative creatinine, preoperative cardiogenic shock, surgical indication), and concomitant medications (angiotensinconverting enzyme inhibitors, clopidogrel, aspirin, $\beta$-blockers, warfarin).

\section{Short-Term and Mid-Term Outcomes}

The primary outcome analyzed was all-cause and cardiac-related mortality, including short-term and late survival (determined from the follow-up data). The prespecified secondary outcomes measured were 30-day morbidity and length of stay (LOS). All outcomes were determined from the standard definitions established by the Society of Thoracic Surgeons database:

1. 30-Day mortality included patients who had died during the hospitalization for the index procedure (even if the visit surpassed 30 days) and those who had died after discharge from the hospital but within 30 days of their surgery.

2. Mid-term survival and the survival interval were ascertained using the Social Security Death Index and retrospective chart review. Patients were documented as alive if they were not listed in the Social Security Death Index at the time of query (June 2009). The cause of death was verified by retrospective database review and was classified as either cardiac or noncardiac related.

3. 30-Day morbidity included myocardial infarction, cerebrovascular accident, cardiac arrest, coma, and major bleeding episodes during the postoperative hospitalization. These morbidity outcomes were analyzed individually and as a composite postoperative endpoint.

4. Two measures of LOS were analyzed: the duration of the use of the intensive care unit (ICU) after surgery and the entire length of the hospital visit from admission to discharge.

Data on the postoperative events and late survival were available for all patients included in the study cohort.

\section{Statistical Analysis}

On the basis of the primary objective of the investigation, the effect of statin therapy in NL and HL patients was analyzed separately. Because HL diac surgery are lacking. We thus sought to elucidate the 
TABLE 1. Demographics and health characteristics

\begin{tabular}{|c|c|c|c|c|c|c|}
\hline \multirow[b]{2}{*}{ Characteristic } & \multicolumn{3}{|c|}{ NL } & \multicolumn{3}{|c|}{ HL } \\
\hline & No statins $(n=1052)$ & Statins $(n=206)$ & $P$ value & No statins $(n=638)$ & Statins $(n=1160)$ & $P$ value \\
\hline \multicolumn{7}{|l|}{ Demographic } \\
\hline Mean age (y) & $57.7 \pm 16$ & $64.1 \pm 12$ & $\leq .0001$ & $64.7 \pm 12$ & $66.0 \pm 11$ & .02 \\
\hline Male & $622(59.1)$ & $151(73.3)$ & $\leq .0001$ & $410(64.3)$ & $824(71.0)$ & .003 \\
\hline \multicolumn{7}{|l|}{ Race/ethnicity } \\
\hline White & $737(70.1)$ & $143(69.4)$ & & $454(71.2)$ & $827(71.3)$ & \\
\hline Black & $102(9.7)$ & $22(10.7)$ & $>.2$ & $58(9.1)$ & $93(8.0)$ & $>.2$ \\
\hline Hispanic/other & $213(20.2)$ & $41(19.9)$ & & $126(19.7)$ & $240(20.7)$ & \\
\hline \multicolumn{7}{|l|}{ Clinical } \\
\hline Body mass index $\left(\mathrm{kg} / \mathrm{m}^{2}\right)$ & $26.8 \pm 6$ & $28.7 \pm 6$ & $\leq .0001$ & $28.2 \pm 6$ & $28.6 \pm 6$ & $>.2$ \\
\hline Current smoker & $271(25.8)$ & 75 (36.4) & .002 & $224(35.1)$ & $390(33.6)$ & $>.2$ \\
\hline Diabetes & $114(10.8)$ & $53(25.7)$ & $\leq .0001$ & $160(25.1)$ & $281(24.2)$ & $>.2$ \\
\hline Hypertension & $444(42.2)$ & $132(64.1)$ & $\leq .0001$ & $461(72.3)$ & 849 (73.2) & $>.2$ \\
\hline $\mathrm{AF}$ & $293(27.9)$ & $38(18.4)$ & .005 & $153(24.0)$ & $210(18.1)$ & .003 \\
\hline $\mathrm{CHF}$ & $316(30.0)$ & $62(30.1)$ & $>.2$ & $220(34.5)$ & $299(25.8)$ & $\leq .0001$ \\
\hline \multicolumn{7}{|l|}{ NYHA class } \\
\hline I & $299(28.4)$ & $40(19.4)$ & & $104(16.3)$ & $279(24.1)$ & \\
\hline II & $397(37.7)$ & $100(48.5)$ & .003 & $231(36.2)$ & $476(41.0)$ & $\leq .0001$ \\
\hline III & $263(25.0)$ & $55(26.7)$ & & $202(31.7)$ & $330(28.4)$ & \\
\hline IV & $92(8.7)$ & $10(4.9)$ & & $101(15.8)$ & $75(6.5)$ & \\
\hline Renal failure & $48(4.6)$ & $9(4.4)$ & $>.2$ & $47(7.4)$ & $60(5.2)$ & .06 \\
\hline Previous dialysis & $23(2.2)$ & $4(1.9)$ & $>.2$ & $21(3.3)$ & $27(2.3)$ & $>.2$ \\
\hline COPD & $94(8.9)$ & $17(8.3)$ & $>.2$ & $94(14.7)$ & $136(11.7)$ & .07 \\
\hline PVD & $54(5.1)$ & $15(7.3)$ & $>.2$ & $57(8.9)$ & $124(10.7)$ & $>.2$ \\
\hline Pulmonary hypertension & & & .08 & & & $\leq .0001$ \\
\hline None & $771(73.3)$ & $138(67.0)$ & & 457 (71.6) & $941(81.1)$ & \\
\hline Moderate $(\geq 35 \mathrm{mmHg})$ & $190(18.1)$ & $51(24.8)$ & & $104(16.3)$ & $153(13.2)$ & \\
\hline Severe $(\geq 50 \mathrm{mmHg})$ & $91(8.7)$ & $17(8.3)$ & & $77(12.1)$ & $66(5.7)$ & \\
\hline Previous MI & $72(6.8)$ & $51(24.8)$ & $\leq .0001$ & $155(24.3)$ & $330(28.4)$ & .06 \\
\hline Previous angina & $167(15.9)$ & $78(37.9)$ & $\leq .0001$ & $215(33.7)$ & $443(38.2)$ & .06 \\
\hline Previous stroke/TIA & $90(8.6)$ & $20(9.7)$ & $>.2$ & $76(11.9)$ & $177(15.3)$ & .05 \\
\hline Family history of CAD & $157(14.9)$ & $37(18.0)$ & $>.2$ & $160(25.1)$ & $291(25.1)$ & $>.2$ \\
\hline
\end{tabular}

Continuous variables are presented as mean \pm standard deviation and were compared between statin users and nonusers using 2-sided Student's independent $t$ test (with Satterthwaite adjustment if variances were unequal). Categorical variables are presented as n (\%) and were compared using $\chi^{2}$ tests. $A F$, Atrial fibrillation; $C H F$, congestive heart failure; NYHA, New York Heart Association; $C O P D$, chronic obstructive pulmonary disease; $P V D$, peripheral vascular disease; $M I$, myocardial infarction; $T I A$, transient ischemic attack; $C A D$, coronary artery disease.

is an established risk factor for adverse cardiac events, we did not compare the HL and NL patient cohorts. The baseline indexes were compared in the statin users versus the statin nonusers (for both NL and HL patients). Continuous variables are presented as the mean \pm standard deviation and were compared using 2-sided Student's independent $t$ tests. The Satterthwaite adjustment was used in cases of unequal variance. The categorical variables are expressed as frequencies and were compared using Pearson's $\chi^{2}$ exact tests. Various multivariate techniques were used to determine the independent contribution of perioperative statin therapy to outcome measures, after adjusting for patient demographics, known preoperative cardiac risk factors, surgical characteristics, and concomitant medications. The primary outcome was analyzed using the Cox proportional hazards model to calculate the hazards ratio (HR) and $95 \%$ confidence interval (CI) to assess the differences in late survival after surgery between the statin users and nonusers and to identify significant independent predictors of mid-term mortality. Schoenfeld residuals, correlated with time and partial residual plots, were used to verify the validity of the proportional hazards assumption. Kaplan-Meier survival curves were constructed and compared using the log-rank test. Odds ratios (ORs) were determined from the logistic regression analyses to compare the postoperative events (30-day morbidity and mortality). Multiple regression analyses were performed to evaluate the effect of statin use on the LOS (ICU and entire hospital visits). Final adjusted models were created using a combination of forward and backward selection procedures. Each baseline covariate listed in Tables 1 and 2 was evaluated individually in single-predictor models for the primary and secondary outcomes. The multivariate models included significant candidate predictors $(P<.2)$ according to this bivariate screening. The final multivariate models for the clinical endpoints were determined by stepwise removal of the variables that were not significant $(P>.05)$. All statistical analyses were performed using STATA statistical software (StataCorp LP, College Station, Tex).

\section{RESULTS}

\section{Baseline Risk Comparisons}

The preoperative baseline characteristics of the 4 groups defined by statin treatment and lipid status are listed in Table 1. Of the $1258 \mathrm{NL}$ patients $(41.1 \%)$ included in the study cohort, the statin users were more likely to be significantly older (by 6.4 years), be male, and have greater rates of obesity and other known cardiac risk factors (ie, 
TABLE 2. Surgical characteristics and concomitant medications

\begin{tabular}{|c|c|c|c|c|c|c|}
\hline \multirow[b]{2}{*}{ Variable } & \multicolumn{3}{|c|}{ NL } & \multicolumn{3}{|c|}{ HL } \\
\hline & No statins $(n=1052)$ & Statins $(n=206)$ & $P$ value & No statins $(n=638)$ & Statins $(n=1160)$ & $P$ value \\
\hline \multicolumn{7}{|l|}{ Surgical characteristics } \\
\hline Ejection fraction $(\%)$ & $53.4 \pm 14$ & $52.7 \pm 13$ & $>.2$ & $50.4 \pm 16$ & $51.9 \pm 14$ & .05 \\
\hline No. of diseased vessels & $0.6 \pm 1$ & $1.8 \pm 1$ & $\leq .0001$ & $1.5 \pm 1$ & $1.9 \pm 1$ & $\leq .0001$ \\
\hline Left main disease & $51(4.8)$ & $42(20.4)$ & $\leq .0001$ & 89 (13.9) & $235(20.3)$ & .001 \\
\hline Preoperative creatinine & $1.1 \pm 1$ & $1.2 \pm 1$ & $>.2$ & $1.3 \pm 1$ & $1.2 \pm 1$ & $>.2$ \\
\hline $\begin{array}{l}\text { Preoperative cardiogenic } \\
\text { shock }\end{array}$ & $38(3.6)$ & $8(3.9)$ & $>.2$ & $32(5.0)$ & $1.2(1.2)$ & $\leq .0001$ \\
\hline \multicolumn{7}{|l|}{ Surgery incidence } \\
\hline First cardiac surgery & $877(83.4)$ & $180(87.4)$ & & 495 (77.6) & $974(84.0)$ & \\
\hline Second cardiac surgery & $150(14.3)$ & $21(10.2)$ & $>.2$ & $116(18.2)$ & $163(14.1)$ & .001 \\
\hline $\begin{array}{l}\text { More than } 2 \text { cardiac } \\
\text { surgeries }\end{array}$ & $25(2.4)$ & $5(2.4)$ & & $27(4.2)$ & $23(2.0)$ & \\
\hline Emergent/urgent & $214(20.3)$ & $48(23.3)$ & $>.2$ & $181(28.4)$ & $259(22.3)$ & .001 \\
\hline \multicolumn{7}{|l|}{ Surgery type } \\
\hline CABG only & $98(9.3)$ & $73(35.4)$ & & $166(26.0)$ & $455(39.2)$ & \\
\hline Valve only & $675(64.2)$ & $57(27.7)$ & $\leq .0001$ & $234(36.7)$ & $320(27.6)$ & $\leq .0001$ \\
\hline $\mathrm{CABG}+$ valve & $121(11.5)$ & $48(23.3)$ & & $145(22.7)$ & $255(22.0)$ & \\
\hline Other cardiac & $158(15.0)$ & $28(13.6)$ & & 93 (14.6) & $130(11.2)$ & \\
\hline \multicolumn{7}{|l|}{ Concomitant medications } \\
\hline ACE inhibitors & $328(31.2)$ & $68(33.0)$ & $>.2$ & $231(36.2)$ & $565(48.7)$ & $\leq .0001$ \\
\hline Clopidogrel & 17 (1.6) & $8(3.9)$ & .03 & $36(5.6)$ & $62(5.3)$ & $>.2$ \\
\hline Aspirin & 228 (21.7) & $98(47.6)$ & $\leq .0001$ & $264(41.4)$ & $584(50.3)$ & $\leq .0001$ \\
\hline$\beta$-Blockers & $494(47.0)$ & $127(61.7)$ & $\leq .0001$ & $371(58.2)$ & $764(65.9)$ & .001 \\
\hline Warfarin & $100(9.5)$ & $10(4.9)$ & .03 & $58(9.1)$ & $69(5.9)$ & .01 \\
\hline
\end{tabular}

Continuous variables are presented as mean \pm standard deviation and were compared between statin users and nonusers using 2-sided Student's independent $t$ test (with Satterthwaite adjustment if variances were unequal). Categorical variables are presented as $\mathrm{n}(\%)$ and compared using $\chi^{2}$ tests. $C A B G$, Coronary artery bypass graft; $A C E$, angiotensin-converting enzyme; $A D P$, adenosine diphosphate.

smoking, diabetes, hypertension). Statin users were more than 3 times as likely to have experienced a previous myocardial infarction and twice as likely to have experienced episodes of angina than the NL population. Statin nonusers were more likely to have greater rates of more advanced New York Heart Association (NYHA) classes and atrial fibrillation. Of the $1798 \mathrm{HL}$ patients (58.9\%), similar, but less pronounced, differences were observed at baseline between the statin users and nonusers. The perioperative statin users were more likely to be older (by 1.3 years), be male and have a had a previous stroke or transient ischemic attach. Also, the HL, statin nonusers were more likely to have an adverse NYHA class distribution, greater rates of congestive heart failure, and more severe pulmonary hypertension. Overall, race, comorbid renal or obstructive lung disease, and family history of coronary disease did not significantly differ across the 4 groups.

The surgical characteristics and concomitant medications of the patients who were and were not pretreated with statins are listed in Table 2. Of the NL patients, statin users were more likely to have a greater number of diseased vessels, left main disease, greater rates of CABG, and to be concomitantly treated with clopidogrel, aspirin, and $\beta$ blockers. In contrast, the NL, statin nonusers were more likely to have undergone more valvular procedures and to receive warfarin therapy. Similar findings were noted in the HL cohort, except that the statin nonusers tended to have more depressed ejection fractions and greater rates of preoperative cardiogenic shock and were more likely to present for repeat or urgent procedures.

\section{Outcomes}

From the adverse clinical risk profiles listed in Tables 1 and 2 , the mortality and morbidity were expected to be worse for the NL statin users. However, despite this, the statin users consistently had better outcomes than the nonusers in this population.

30-day mortality. For the NL cohort, statin therapy effectively reduced the 30 -day mortality rate to $0 \%(0 /$ 206) for the NL patients taking statins compared with $3.0 \%(32 / 1052)$ for the NL statin nonusers $(P=.01$, Table 3).

For the HL cohort, as expected, perioperative statin therapy also significantly reduced the 30-day mortality rate $(8.0 \%$ [51/638] vs $0.7 \%$ [8/1160]), with a univariate OR of 0.08 (95\% CI, 0.04-0.17; $P \leq .0001)$. These differences in early mortality persisted even after adjusting for the significant baseline covariates (adjusted OR, 0.11; 95\% CI, $0.05-0.25 ; P \leq .0001)$, strongly favoring perioperative statin therapy (Table 3 ). 
TABLE 3. Univariate and multivariate logistic regression analyses for 30-day morbidity and mortality in normolipidemic and hyperlipidemic patients undergoing cardiac surgery

\begin{tabular}{|c|c|c|c|c|c|c|}
\hline Variable & No statin use & Statin use & Univariate OR $(95 \%$ CI $)$ & $P$ value & Risk-adjusted OR $(95 \%$ CI $)$ & $P$ value \\
\hline NL patients & $1052(100)$ & $206(100)$ & & & & \\
\hline 30-d Mortality & $32(3.0)$ & $0(0.0)$ & - & .01 & - & - \\
\hline 30-d Combined morbidity & $60(5.7)$ & $4(1.9)$ & $0.33(0.12-0.91)$ & .03 & $0.26(0.09-0.80)$ & .02 \\
\hline MI & $14(1.3)$ & $1(0.5)$ & $0.36(0.05-2.77)$ & $>.2$ & $0.16(0.02-1.49)$ & .11 \\
\hline CVA & $18(1.7)$ & $2(1.0)$ & $0.56(0.13-2.44)$ & $>.2$ & $0.66(0.13-3.35)$ & $>.2$ \\
\hline Cardiac arrest & $32(3.0)$ & $1(0.5)$ & $0.16(0.02-1.14)$ & .07 & $0.10(0.01-0.83)$ & .03 \\
\hline Coma & $7(0.7)$ & $0(0.0)$ & - & $>.2$ & - & - \\
\hline Bleeding & $7(0.7)$ & $0(0.0)$ & - & $>.2$ & - & - \\
\hline HL patients & $638(100)$ & $1160(100)$ & & & & \\
\hline 30-d Mortality & $51(8.0)$ & $8(0.7)$ & $0.08(0.04-0.17)$ & $\leq .0001$ & $0.11(0.05-0.25)$ & $\leq .0001$ \\
\hline 30-d Combined morbidity & $54(8.5)$ & $54(4.7)$ & $0.53(0.36-0.78)$ & .001 & $0.60(0.39-0.92)$ & .02 \\
\hline MI & $8(1.3)$ & $16(1.4)$ & $1.10(0.47-2.59)$ & $>.2$ & $0.93(0.38-2.25)$ & $>.2$ \\
\hline CVA & $17(2.7)$ & $19(1.6)$ & $0.58(0.29-1.13)$ & .11 & $0.76(0.35-1.65)$ & $>0.2$ \\
\hline Cardiac arrest & $22(3.4)$ & $18(1.6)$ & $0.44(0.23-0.83)$ & .01 & $0.10(0.01-0.83)$ & .03 \\
\hline Coma & $7(1.1)$ & $1(0.1)$ & $0.08(0.01-0.63)$ & .02 & $0.07(0.01-0.69)$ & .02 \\
\hline Bleeding & $10(1.6)$ & $5(0.4)$ & $0.27(0.09-0.80)$ & .02 & $0.39(0.12-1.28)$ & .12 \\
\hline
\end{tabular}

Outcome variables are presented as n (\%).P values were determined by univariate and multivariate logistic regression analyses, except for outcomes with no events (which were compared using Pearson's $\chi^{2}$ tests). NL, Normolipidemic; $C I$, confidence interval; OR, odds ratio; $M I$, myocardial infarction; $C V A$, cerebrovascular accident.

Late survival. The mean follow-up for late survival was $26.2 \pm 16.9$ months. The predictors of late survival in the final model were distinct for the NL and HL groups.

For the NL cohort, the overall all-cause mortality rate was $9.6 \%$ for the statin-untreated group and only $3.9 \%$ in the statin-treated group. Univariate analysis of the primary outcome showed that statin therapy significantly reduced mid-term mortality (HR, $0.40 ; 95 \%$ CI, 0.19-0.82; $P=.008)$. The strength of the association became even stronger after adjusting for the adverse risk factors in the statin group. Perioperative statin therapy independently reduced the mid-term mortality (adjusted HR, $0.34 ; 95 \%$ CI, $0.16-0.71 ; P=.004$; Figure $1, A$ ). Statin users also experienced lower rates of cardiac death $(3.3 \%$ vs $0.5 \%)$ and noncardiac death $(6.3 \%$ vs $3.4 \%)$ when analyzed separately. The significant reductions in cause-specific death were independent of the underlying baseline risk indexes. The riskadjusted HR was $0.14(95 \%$ CI, $0.02-1.00 ; P=.05)$ for cardiac-related mortality and $0.43(95 \% \mathrm{CI}, 0.19-0.98$; $P=.05$ ) for noncardiac-related mortality (Figure 1, $A$ ).

Figure 2, $A$ shows the significant predictors of all-cause mid-term mortality in the fully adjusted Cox proportional hazards model. For NL patients, perioperative statin therapy and body mass index remained protective factors against the primary outcome. In contrast, perioperative warfarin therapy, combination valve/CABG, advanced NYHA classes, smoking, and age all increased the risk of all-cause mortality.

For the HL cohort, statin therapy in the perioperative period of cardiac surgery reduced the overall rate of all-cause mortality from $17.2 \%$ for nonusers to $6.5 \%$ for statin users. Statin users had consistently favorable mid-term mortality outcomes compared with their nonuser counterparts. The adjusted HR was 0.42 (95\% CI, 0.31-0.57; $P \leq .0001)$ for all-cause mortality, 0.16 (95\% CI, 0.08-0.34; $P \leq .0001)$ for cardiac-related death, and 0.52 (95\% CI, 0.36-0.73; $P \leq .0001$ ) for noncardiac-related death, robustly supporting perioperative statin use for HL patients (Figure 1, $B$ ).

Figure 2, $B$ presents the significant independent predictors of late survival for HL patients. Perioperative statin therapy and gender (males) were inversely associated with all-cause mortality. Perioperative warfarin therapy, a repeat or an urgent procedure, preoperative cardiogenic shock, preoperative creatinine level, severe pulmonary hypertension, advanced NYHA classes, and age all increased the risk of death after cardiac surgery.

Finally, the Kaplan-Meier survival curves showed early and continued separation of the mortality trends throughout the follow-up period $(P \leq .0001)$ (Figure 3). Statin use, regardless of baseline lipid status, improved late survival. HL and NL patients who were not pretreated with statins had increased mortality compared with the 2 groups receiving statin therapy.

30-day morbidity. Table 3 lists the postoperative nonfatal adverse events for the NL and HL patients. Statin therapy consistently reduced all major surgical complications for NL patients, with a composite 30-day morbidity rate of $5.7 \%(60 / 1052)$ for nonusers compared with only $1.9 \%$ (4/206) for statin users. Statin users did not experience any coma or bleeding events in the early postoperative period. The risk-adjusted OR for 30-day morbidity was 0.26 (95\% CI, 0.09-0.80; $P=.02$ ).

Statin therapy for the HL cohort also reduced a wide range of major complications after cardiac surgery, with a composite 30-day morbidity rate of $8.5 \%(54 / 638)$ for nonusers compared with almost one half that rate for statin users $(4.7 \% ; 54 / 1160)$. Perioperative statin therapy independently 

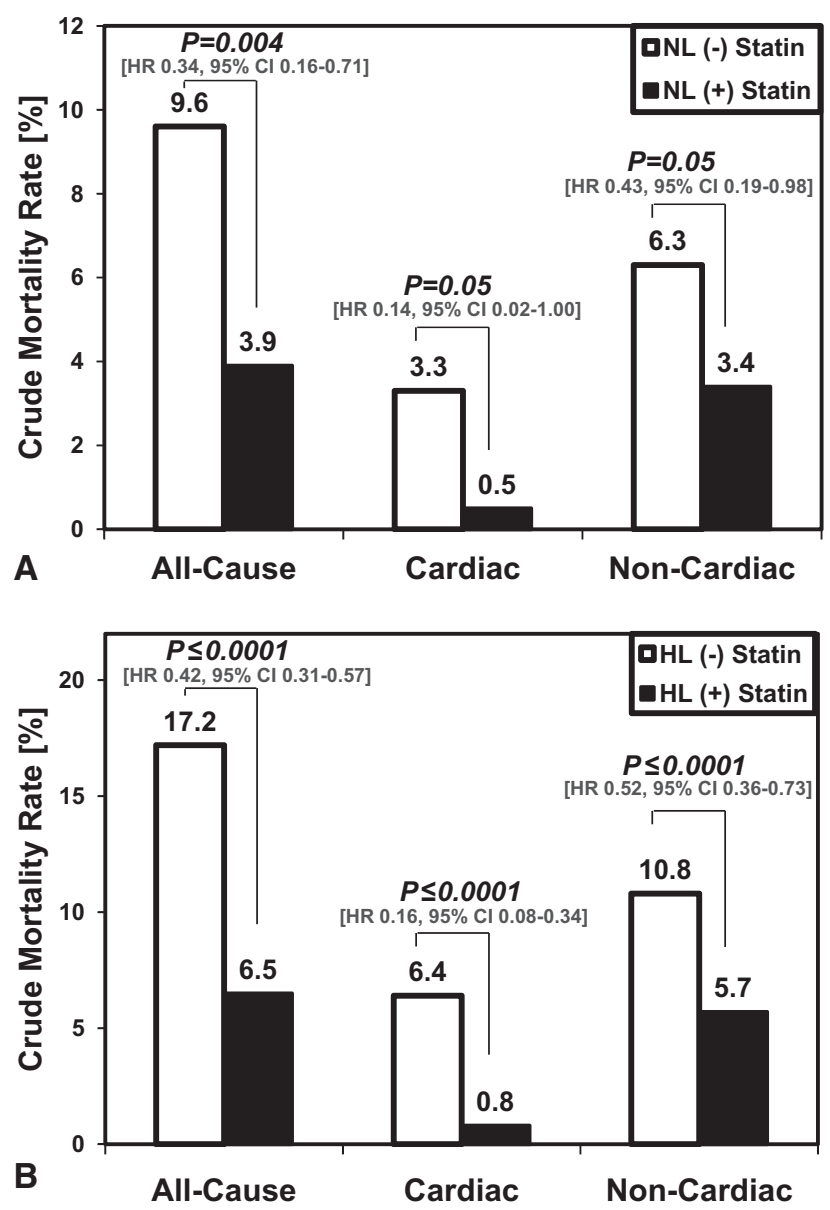

FIGURE 1. Multivariate hazard ratios for primary outcome (late survival) in A, normolipidemic $(N L)$ and B, hyperlipidemic $(H L)$ patients undergoing cardiac surgery. HR, Hazard ratio; (-) statin, no statin therapy; (+) statin, statin therapy.

reduced the rates of 30-day morbidity events, with an adjusted OR of 0.60 (95\% CI, 0.39-0.92; $P=.02)$.

Length of stay (Figure 4). In the NL cohort, multiple regression analysis demonstrated that statin therapy reduced the LOS of the ICU visit and the total hospital visit. Despite showing a trend in the right direction, the riskadjusted $\beta$ coefficients were not significant.

In the HL cohort, statin therapy strongly reduced the ICU component of the LOS by 1.6 days $(95 \% \mathrm{CI},-2.3$ to -0.9 ; $\beta$ coefficient, $-0.11 ; P \leq .0001)$ and the LOS of the entire hospital visit by 1.7 days $(95 \% \mathrm{CI},-2.7$ to $-0.8 ; \beta$ coefficient, $-0.07 ; P \leq .0001)$.

\section{DISCUSSION}

This is first study to demonstrate that perioperative statin therapy significantly reduces early, as well as late, cardiovascular events after cardiac surgery in NL patients. Statin therapy in our study was shown to protect against 30-day mortality, 30-day composite morbidity, and mid-term mor-
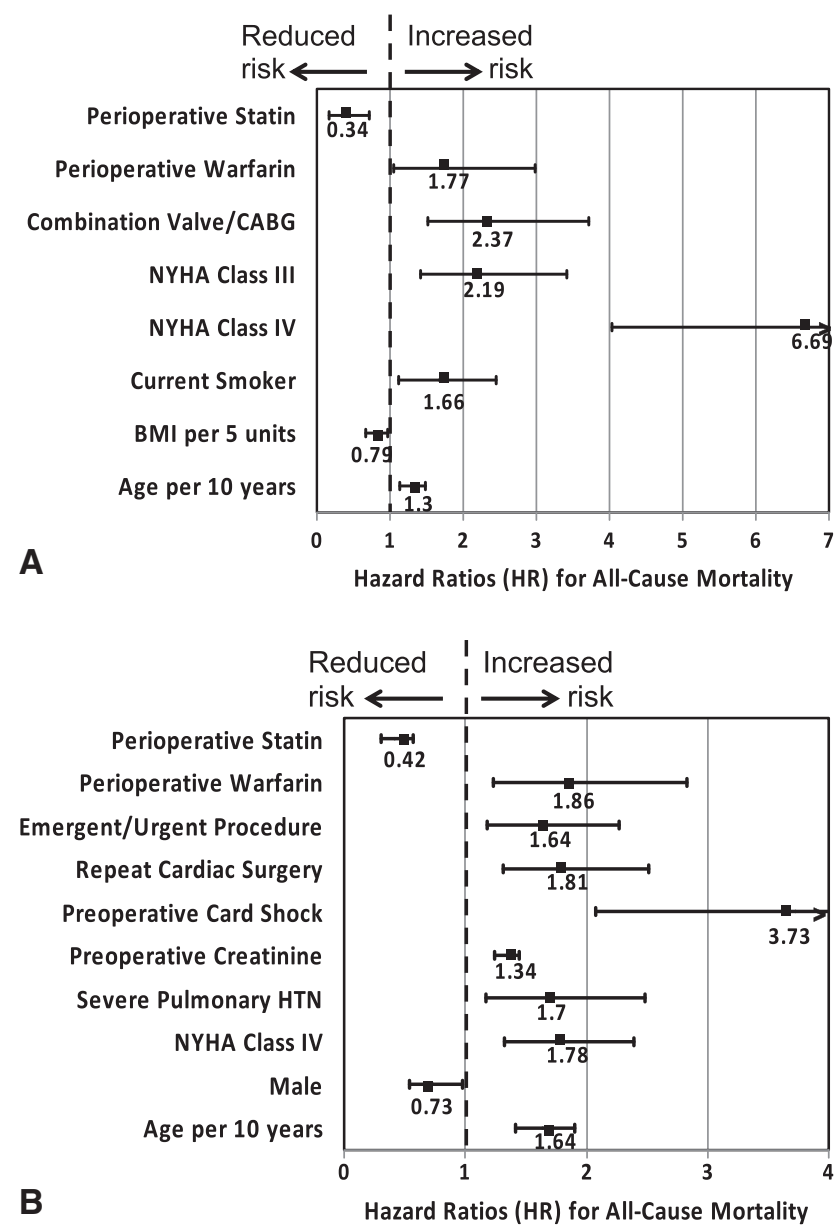

FIGURE 2. Significant predictors of primary outcome (late survival, allcause mortality) in final Cox proportional hazards models in A, normolipidemic and $\mathrm{B}$, hyperlipidemic patients undergoing cardiac surgery. $C A B G$, Coronary artery bypass graft; NYHA, New York Heart Association; BMI, body mass index; HTN, hypertension.

tality (all-cause, cardiac, and noncardiac mortality), independently of the known preoperative cardiac risk factors. Statin therapy reduced the 30-day mortality rate among NL patients to $0 \%$ and more than halved the mid-term mortality. Although statins reduced the postoperative LOS by approximately 1 day, these results were not significant. Because statin therapy has been previously demonstrated to be effective in patients with abnormal lipid profiles, the inclusion of HL patients in our analytic cohort served as an internal control. As expected, perioperative statin therapy for HL patients strongly reduced the incidence of short- and midterm adverse outcomes. Also, statin therapy reduced the LOS during the ICU visit and the total hospital visit in this population by more than 1.5 days. The latter finding might be particularly important in the current healthcare environment. Elevated LDL cholesterol levels have long been recognized as an important risk factor for future cardiovascular events and a worse prognosis after coronary 


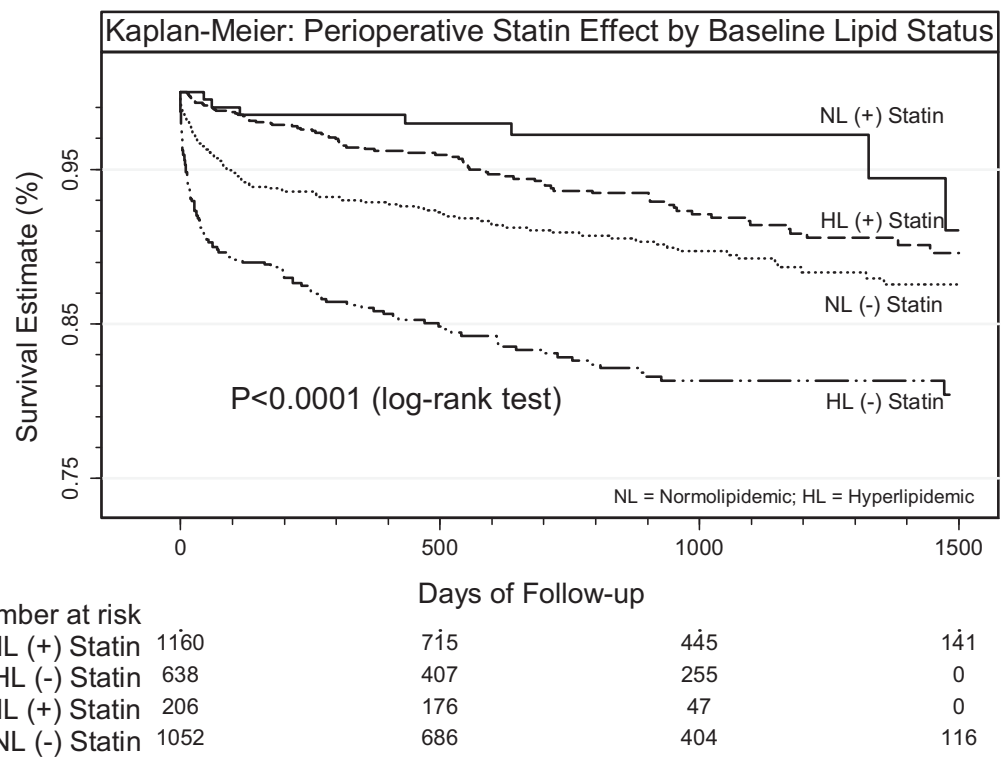

FIGURE 3. Kaplan-Meier survival curves for normolipidemic patients not receiving statin therapy $[N L(-)]$, normolipidemic patients taking statins $[N L(+)]$, hyperlipidemic patients not treated with statins $[H L(-)]$ and hyperlipidemic patients treated with statins $[H L(+)]$.

interventions. ${ }^{21}$ It was thus surprising that the assessment of long-term survival up to a mean follow-up of 2.2 years showed that statin users had superior survival, regardless of their baseline lipid status. These results support the initiation of aggressive lipid-lowering therapy in patients undergoing cardiac surgery, without a primary regard of the lipid status.

These findings from patients undergoing cardiac surgery are informative for many reasons. A substantial number of studies have supported perioperative treatment of patients undergoing $\mathrm{CABG} .{ }^{22} \mathrm{~A}$ recent meta-analysis, summarizing the findings from 19 studies (including 3 randomized controlled trials), confirmed the beneficial effects of statin therapy for this surgical population from the pooled data. ${ }^{5}$ It is difficult, however, to determine whether this apparent cardioprotection is garnered from lipid-dependent or lipidindependent effects. Thielmann and colleagues ${ }^{6}$ were the first to attempt to clinically approach this issue by assessing

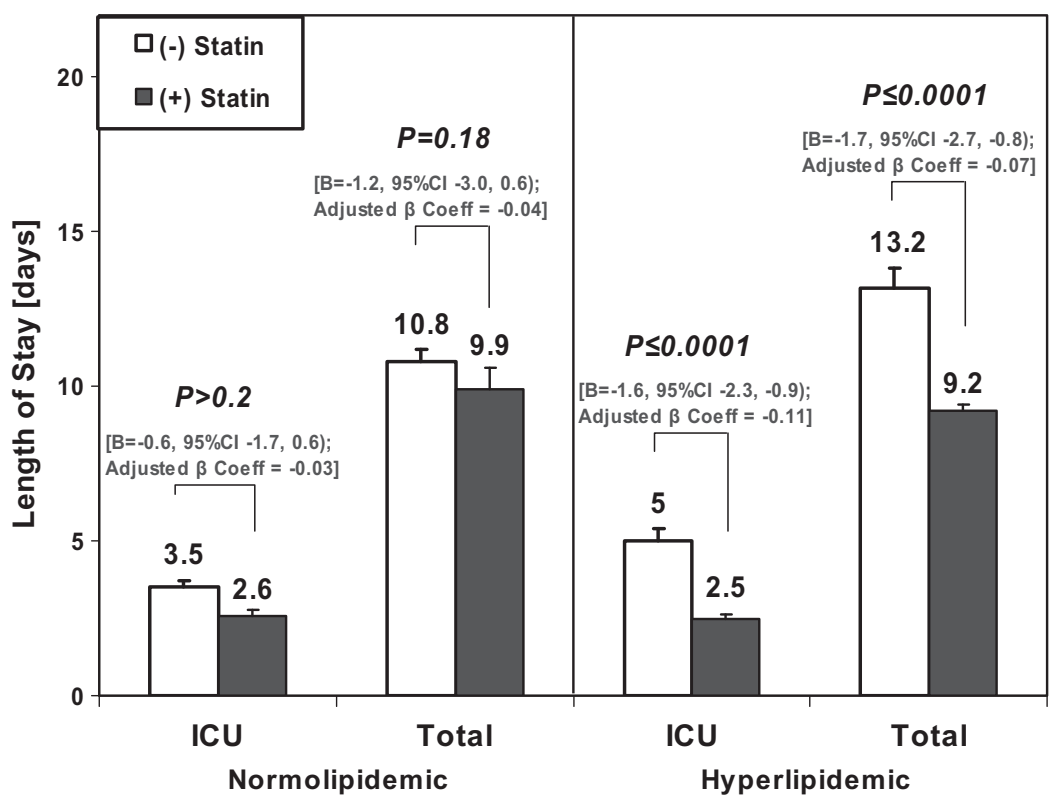

FIGURE 4. Multiple regression analyses predicting length of stay in intensive care unit (ICU) and total hospital visit for normolipidemic and hyperlipidemic patients undergoing cardiac surgery. 
the statin treatment effects on short-term outcomes among HL patients. The investigators defined HL similar to the definition used in our study, but they defined all patients who had had pretreatment with statins as having had abnormal lipid profiles. Since the publication of their work in a previous issue of the Journal, ${ }^{6}$ the indications for statin therapy have continued to expand. We began to observe a previously unrecognized emergent subset population in our surgical series of patients. NL patients who were perioperatively treated with statins represented a relatively small fraction $(16.4 \%)$ of all NL patients presenting for cardiac surgery in our study. These patients, however, experienced the best outcomes across all groups analyzed, despite being older and having greater rates of comorbid disease. Our unique study design that included 4 groups of patients stratified by baseline lipid level and treatment status allowed for a more definitive approach to the question of LDL-independent (pleiotropic) and LDL-dependent statin actions.

Although we were unable to mechanistically assess the effects of statin therapy on the cardiac and systemic biology, our results have provided clinical data that cardioprotection with statins extends beyond their lipid-lowering capacity. The results from our surgical series have demonstrated an early, pronounced reduction in events that might not be adequately explained by the alterations in cardiac risk conferred by LDL reduction alone. Surgery requiring cardiopulmonary bypass acutely creates a pro-inflammatory state, with accompanying marked myocardial necrosis. ${ }^{23,24}$ Statins might play a unique role in modulating this inflammatory milieu, tipping the balance in favor of recovery. ${ }^{7-10}$ Furthermore, the therapeutic effects of statins might continue to be apparent even outside the operating room. Some of the effects not directly related to LDL reduction include physiologic changes in endothelial function, coagulation, platelet activation, smooth muscle responsiveness, inflammatory cell function, and thrombus stability. ${ }^{16,17}$ Our findings of early and late reduction in morbidity and mortality with statin therapy could be explained by these pleiotropic effects.

A few key limitations should be recognized regarding the present study. First and foremost, a major limitation was the retrospective, nonrandomized study design. Considerable baseline differences were present between the statin users and nonusers that made our final interpretation of the study endpoints difficult. Although we performed extensive risk adjustment to account for these vast differences in the baseline characteristics, confounders that were not recorded might have continued to influence our primary and secondary outcomes. For instance, we did not have reliable, validated measures of socioeconomic status included in our current database. Furthermore, statin therapy might have been a surrogate for greater health-seeking behavior in general. The NL statin users were more likely to be concomitantly treated with clopidogrel, aspirin, and $\beta$-blockers than were the nonusers. Additionally, statins appeared to significantly reduce, not only cardiac, but also noncardiac, mortality in our cohort, supporting this "healthy-user effect." However, despite recording a history of hyperlipidemia within 6 months of the index surgery, data regarding the LDL cholesterol levels (as a continuous variable) before surgery were not available. Such data would have allowed for more robust analysis of the relationship between LDL cholesterol reduction and event rates after cardiac surgery. Another major limitation of our study was that the precise reasons behind withholding statin therapy in this patient population were not explicitly determined. The baseline patient characteristics (eg, elevated liver enzymes) related to these treatment differences might have influenced the shortand long-term cardiovascular outcomes. Finally, our institutional database did not include data detailing the specific dosages, types of statins (high or low efficacy), or durations of therapy. Our hypothesis-generating work warrants additional investigation into the role of statin therapy for patients with normal baseline lipid levels presenting for cardiovascular surgery. Large prospective studies are warranted to help establish guidelines for appropriate targets for therapy (dosing, type, and duration of use) for a given baseline risk factor profile.

Despite great strides in perioperative care and improvements in surgical technique, patients undergoing cardiac surgery remain at substantial risk of early and late cardiovascular complications. This risk is especially striking in patients with underlying comorbid conditions and known cardiac risk factors, including hyperlipidemia. Statin therapy might help attenuate the excess risk conferred by high LDL cholesterol before cardiac surgery. Thus, HL patients taking statins might have similar (or even better) prognoses compared with untreated NL patients. Patients undergoing cardiac surgery should not be categorically included or excluded from the benefits of powerful lipidlowering therapy. Despite mounting evidence supporting the use of statin therapy in the setting of cardiac surgery, statins remain considerably underused in the perioperative period. $^{25}$ Our findings have important implications for treatment targets, perioperative patient management, and risk stratification in the setting of cardiac surgery. The results of the present study have changed our practice to a more aggressive use of statins in cardiac surgery, regardless of the patients' preoperative cholesterol levels. Future studies will determine whether this change is warranted for the entire cardiac surgery population.

We would like to thank Michael Yensel, Anna Huskin, and the Bluhm Cardiovascular Institute Clinical Trials Unit for their support and excellent technical assistance.

\footnotetext{
References

1. Goldstein JL, Brown MS. The LDL receptor. Arterioscler Thromb Vasc Biol. 2009;29:431-8.
} 
2. Mills EJ, Rachlis B, Wu P, Devereaux PJ, Arora P, Perri D. Primary prevention of cardiovascular mortality and events with statin treatments: A network metaanalysis involving more than 65,000 patients. J Am Coll Cardiol. 2008;52: 1769-81.

3. Wilt TJ, Bloomfield HE, MacDonald R, Nelson D, Rutks I, Ho M, et al. Effectiveness of statin therapy in adults with coronary heart disease. Arch Intern Med. 2004;164:1427-36.

4. Grundy SM, Cleeman JI, Merz CN, Brewer HB Jr, Clark LT, Hunninghake DB, et al. Implications of recent clinical trials for the National Cholesterol Education Program Adult Treatment Panel III guidelines. Circulation. 2004;110:227-39.

5. Liakopoulos OJ, Choi YH, Haldenwang PL, Strauch J, Wittwer T, Dorge H, et al. Impact of preoperative statin therapy on adverse postoperative outcomes in patients undergoing cardiac surgery: A meta-analysis of over 30,000 patients. Eur Heart J. 2008;29:1548-59.

6. Thielmann M, Neuhauser M, Marr A, Jaeger BR, Wendt D, Schuetze B, et al. Lipid-lowering effect of preoperative statin therapy on postoperative major adverse cardiac events after coronary artery bypass surgery. J Thorac Cardiovasc Surg. 2007; 134:1143-9.

7. Martinez-Comendador JM, Alvarez JR, Mosquera I, Sierra J, Adrio B, Carro JG, et al. Preoperative statin treatment reduces systemic inflammatory response and myocardial damage in cardiac surgery. Eur J Cardiothorac Surg. 2009;36:998-1005.

8. Morgan C, Zappitelli M, Gill P. Statin prophylaxis and inflammatory mediators following cardiopulmonary bypass: A systematic review. Crit Care. 2009;13: R165.

9. Lazar HL, Bao Y, Zhang Y, Bernard SA. Pretreatment with statins enhances myocardial protection during coronary revascularization. J Thorac Cardiovasc Surg. 2003; $125: 1037-42$.

10. Mannacio VA, Iorio D, De Amicis V, Di Lello F, Musumeci F. Effect of rosuvastatin pretreatment on myocardial damage after coronary surgery: A randomized trial. J Thorac Cardiovasc Surg. 2008;136:1541-8.

11. Albert MA, Danielson E, Rifai N, Ridker PM. Effect of statin therapy on Creactive protein levels: The pravastatin inflammation/CRP evaluation (PRINCE): A randomized trial and cohort study. JAMA. 2001;286:64-70.

12. Ridker PM, Rifai N, Pfeffer MA, Sacks F, Braunwald E, The Cholesterol and Recurrent Events (CARE) Investigators. Long-term effects of pravastatin on plasma concentration of C-reactive protein. Circulation. 1999;100:230-5.

13. Pai JK, Pischon T, Ma J, Manson JE, Hankinson SE, Joshipura K, et al. Inflammatory markers and the risk of coronary heart disease in men and women. $N$ Engl J Med. 2004;351:2599-610.

14. Ridker PM, Hennekens CH, Buring JE, Rifai N. C-reactive protein and other markers of inflammation in the prediction of cardiovascular disease in women. N Engl J Med. 2000;342:836-43.

15. Ridker PM, Danielson E, Fonseca FA, Genest J, Gotto AM Jr, Kastelein JJ, et al. Rosuvastatin to prevent vascular events in men and women with elevated Creactive protein. N Engl J Med. 2008;359:2195-207.

16. Davignon J. Beneficial cardiovascular pleiotropic effects of statins. Circulation. 2004;109(23 Suppl. 1):III39-43

17. Liao JK. Effects of statins on 3-hydroxy-3-methylglutaryl coenzyme A reductase inhibition beyond low-density lipoprotein cholesterol. Am J Cardiol. 2005;96: 24F-33.

18. Tabata M, Khalpey Z, Cohn LH, Chen FY, Bolman RM III, Rawn JD. Effect of preoperative statins in patients without coronary artery disease who undergo cardiac surgery. J Thorac Cardiovasc Surg. 2008;136:1510-3.

19. Fedoruk LM, Wang H, Conaway MR, Kron IL, Johnston KC. Statin therapy improves outcomes after valvular heart surgery. Ann Thorac Surg. 2008;85:1521-6.

20. Stone NJ. Stopping statins. Circulation. 2004;110:2280-2.

21. Kannel WB, Dawber TR, Kagan A, Revotskie N, Stokes J III. Factors of risk in the development of coronary heart disease-Six year follow-up experience: The Framingham study. Ann Intern Med. 1961;55:33-50.

22. Takagi H, Kawai N, Umemoto T. Preoperative statin therapy reduces postoperative all-cause mortality in cardiac surgery: A meta-analysis of controlled studies. J Thorac Cardiovasc Surg. 2009;137:e52-3.

23. Czerny M, Baumer H, Kilo J, Lassnigg A, Hamwi A, Vukovich T, et al. Inflammatory response and myocardial injury following coronary artery bypass grafting with or without cardiopulmonary bypass. Eur J Cardiothorac Surg. 2000;17: 737-42.

24. Wan S, Yim AP. Cytokines in myocardial injury: Impact on cardiac surgical approach. Eur J Cardiothorac Surg. 1999;16(Suppl 1):S107-11.

25. Kulik A, Levin R, Ruel M, Mesana TG, Solomon DH, Choudhry NK. Patterns and predictors of statin use after coronary artery bypass graft surgery. $J$ Thorac Cardiovasc Surg. 2007;134:932-8.

\section{Discussion}

Dr David A. Fullerton (Aurora, Colo). Dr Lee, I enjoyed your presentation very much, and I think it is important that we in the audience acknowledge that it is not necessarily easy to (a) collate the information, the data on such a cohort of patients, and then (b) try to drill down and actually make sense of it, so I think you and your group are to be congratulated on that.

Having said that, I think it is very important, as you have done, to acknowledge the limitations of such studies. It is a very heterogeneous group of patients. You appropriately acknowledge the many recognized and unrecognized confounding variables that may have influenced the data. Having said that, just as you accurately pointed out, the prevailing literature strongly supports the notion that there seems to be an association with statin usage around the time of a variety of different types of cardiac operations and favorable outcomes. That said, I wanted to invite you to speculate a little bit on how the mechanism of such an association might be explored. For instance, I think it would be a pretty straightforward matter to take blood or tissue samples seeking evidence of proinflammatory stimulation or anti-inflammatory effects of statins, that sort of thing. I was curious-have you and your group discussed this? Have you embarked on this, or would that be of interest to you?

Again, I enjoyed your talk very much and thank you.

Dr Lee. Thank you for those comments and the questions.

I agree. We have one of a few different ways that we are sorting out where we go to next. Clearly we need to do this in a more prospective fashion and we need to look a little bit better at the patients who were not started preoperatively for 6 months but were started postoperatively and what happens to them longterm as well. So there are still some things within the data set that we need to capture more effectively; however, in the next step, we do need to look at troponins, CK MBs, and CRPs around the time of surgery in the users versus nonusers, and do a much more sophisticated analysis of that patient population. I think what really needs to be done is a much bigger prospective study on a wider scale. Again, and I appreciate your comments-this is the beginning and it may or may not be real but I know I am taking an aspirin a day and that was based on an early study a long time ago. We may evolve to the point where we start taking a statin a day, too.

Dr Irving Kron (Charlottesville, Va). Dr Lee, I enjoyed your talk. We have published similar data just a couple of years ago and, in fact, we had proposed-as you all know, there is a network at the NHLBI doing cardiovascular surgical research and this is one study we proposed in valvular heart disease. I think it is a real thing and there are data at least in animals, Dr. Fullerton, that looks at these issues, so you have convincing data. Are you going to have every valve patient that comes in started on a statin?

Dr Lee. We already do. The question is how to get them 6 months before. I think we need to do again is if this gets a little more weight we need to not only present it at a forum like this. We had an offshoot of this presentation that we presented at the ACC but we need to help our cardiology colleagues look at this issue as well.

Dr John C. Chen (Honolulu, Hawaii). As an extension of that, how long in advance would you have them start on the statin prior to surgery? 
Dr Lee. The answer is yes. [Laughter] I think realistically that is what the trial would be for and as surgeons-I don't know about your practice but a lot of my practice is in-house, so I think at time of diagnosis but if you really want to look at it, you look at your valve practice. Take the valve people. You could probably hold off at least 2 to 4 weeks on the valve and design it that way. Otherwise, it actually probably is not very good because if you have to wait too long for the valve population this kind of stuff is not good. This won't help them at all unless even that 2-month window makes a difference and that is why we need to start with a trial.

Dr Craig Baker (Los Angeles, Calif). Thank you for an excellent presentation. As we heard a couple days ago, there is very convincing evidence that children with congenital heart disease are becoming a large growing population and we are increasingly operating on teenagers and young adults with tetrology repairs, putting in, as we heard, bioprosthetic valves. This may require a little bit of speculation but what would be your advice in this young population who are having new valves put in as many of them are elective and we would have time to start preoperative therapy. It is not usually in an urgent setting. In fact, I was rounding last week and one of our 25-year-olds who we did this procedure in and the team had started them on statin therapy and we at rounds said, hey, we don't want to put this young patient on medication he may take for the next 30 to 40 years. Could you offer any advice for this particular patient population?

Dr Lee. As you know, the recommendation from the ACCHA was to start it even in the teen population, which was a little controversial, but we also have done an analysis of purely valve patients looking at users, nonusers, stoppers, and starters. In fact, we found a survival advantage for those patients who were put on statin therapy and continued on it even when it was postoperative. When patients stopped it for any reason whatsoever they had a survival disadvantage. Again, that's cloudy. Who knows why they stopped it? But at least it is our preliminary impression that, yes, patients undergoing valve surgery, particularly with bioprosthetic valves, should be getting statins. We need more literature to really support that but that would be our practice in the current era. 